\title{
Impact of microstructure on the electrical properties of zirconium titanate thin films in MOS configuration
}

\author{
P.Victor ${ }^{1}$, J.Nagaraju ${ }^{2}$ and S.B.Krupanidhi ${ }^{1}$ \\ 'Materials Research Center, Indian Institute of Science, Bangalore, India \\ ${ }^{2}$ Department of Instrumentation, Indian Institute of Science, Bangalore, India \\ victor@mrc.iisc.ernet.in
}

\begin{abstract}
Zirconium titanate thin films were deposited on $\mathrm{p}$ - type Si substrate by pulsed excimer laser ablation technique. The thin films were highly oriented along (020) and polycyrstalline depending on the deposition parameters. A correlation of the microstructure with the electrical properties has been studied in detail. C - V, G $\mathrm{V}$ and DC leakage current studies were done on both types of the films. The higher dielectric constant and higher leakage current were observed on the highly oriented thin films than the polycrystalline thin films. The $\mathrm{C}-\mathrm{V}$ and $\mathrm{G}-\mathrm{V}$ measurements were carried at higher frequency ranging from $1 \mathrm{kHz}$ to $100 \mathrm{kHz}$ in elevated temperatures and the activation energy calculated from arrhenius plot were approximately half the silicon band gap. This suggests that generation recombination mechanism is taking place in the depletion region of Si substrate through bulk traps. The interface states were calculated using high frequency method and it were higher for the polycrystalline than highly oriented thin films. The electronic conduction mechanism is analysed on both the types of films in detail, applying different kinds of mechanism like poole frenkel, schottky and Space charge limited conduction
\end{abstract}

\section{INTRODUCTION}

High dielectric constant materials are of great interest in many integrated devices such as dynamic random access memory (DRAM), [1] gate dielectrics and microwave dielectrics[3]. Miniaturization is the key for the microelectronics, hence, there is a constant surge for the alternative of $\mathrm{SiO}_{2}$ dielectric thin films to replace with other higher dielectric constant materials such that it exhibits excellent thermal stability, lattice match of the Si substrate and lower dielectric loss.

Recently, the perovskite materials like $(\mathrm{Ba}, \mathrm{Sr}) \mathrm{TiO}_{3}, \mathrm{SrTiO}_{3}$ thin films [4] and linear dielectrics like $\mathrm{TiO}_{2}$, [5] $\mathrm{ZrTiO}_{4}$ and $\mathrm{Sn}$ modified $\mathrm{ZrTiO}_{4}$ are been extensively investigated. $[3,6]$. Most of these materials finds usefulness in gate dielectrics and microwave applications due to their higher dielectric constant, high $Q$ factor and lower temperature coefficient of resonance. Unfortunately most of the materials are not thermally stable on $\mathrm{Si}$, hence requires a passivation layer, in order to avoid the formation of metal silicides or diffusion into
$\mathrm{Si}$, thereby resulting in the degradation of the device performance. Hence a correlation of the electrical properties with the microstructure of thin films is essential, as the morphology of the thin films is vital for the best performance of the device.

\section{EXPERIMENT}

Highly oriented and polycrystalline zirconium titanate thin films was deposited on a $p$ type Si substrate using pulsed excimer laser ablation technique $(\mathrm{KrF}, 248 \mathrm{~nm})$ from a single phase $\mathrm{ZrTiO}_{4}$ target. $\mathrm{ZrTiO}_{4}$ target was prepared via conventional solid - state reaction and the films was deposited at a substrate temperature from $300^{\circ} \mathrm{C}$ to $650^{\circ} \mathrm{C}$ with an operating pressure at $5-100$ mtorr in $100 \%$ oxygen ambient. All the as - grown films below $400^{\circ} \mathrm{C}$ were amorphous, hence these thin films was annealed using conventional furnace annealing for $45 \mathrm{mts}$ from $700-775^{\circ} \mathrm{C}$ to induce crystallinity. [7]

The thickness of the ZT thin films was 150 $-300 \mathrm{~nm}$ and was measured by thin film thickness monitor system (FILMETRICS). Gold dots of area $\left(1.96 \times 10^{-3} \mathrm{~cm}^{2}\right)$ was deposited by thermal evaporation on the $\mathrm{ZT}$ thin films for the metal insulator - semiconductor configuration to carry out the electrical measurement. The dielectric measurement was done using a Keithley LCZ 3330 coupled with Keithley SMU 236 to do C - V measurement. The dc leakage current was measured using a computer interfaced Keithley programmable SMU 236. All the measurements was carried at different temperatures from $30^{\circ} \mathrm{C}$ to $250^{\circ} \mathrm{C}$ on the ZT thin films.

\section{RESULTS AND DISCUSSION}

\section{Structure}

Figure 1 shows the XRD patterns of the ZT target and thin films. The thin films deposited insitu at higher substrate temperature of $550^{\circ} \mathrm{C}$, was of highly oriented along (020) direction and the films grown ex-situ at $300^{\circ} \mathrm{C}$ was amorphous and on subsequent annealing resulted in polycrystalline thin 
films. The polycrystalline ZT thin films exhibited the dominance of (111) peak over (020). Highly oriented ZT thin films show a (020) fiber texture and the polycrystalline ZT thin films are randomly oriented along (011), (111), (020).

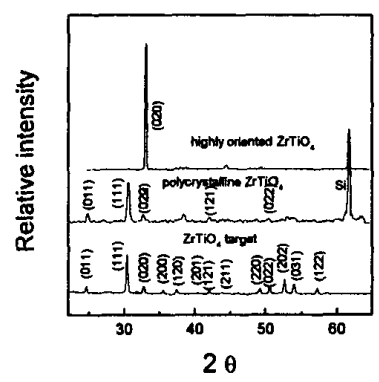

Figure 1. X - ray diffraction of highly oriented and polycrystalline ZT thin films.

The composition analysis was done using energy dispersive analysis (EDX) and the films maintained the $\mathrm{Zr} / \mathrm{Ti}$ ratio of 0.9 to 1.1 . The thin films grown at lower substrate temperatures possess low adatom mobility conditions, eventually the microstructure will be fine grained. During post - deposition annealing, these thin films undergo a change in the microstructure due to thermally induced nucleation and grain growth. The amorphous thin films with its inhomogenous nucleation and subsequently grain growth due to annealing will result in random polycrystalline grains in the thin films. According to structure - zone model (SZM) proposed by Thorton, at higher substrate temperatures, the microstructure was found to undergo a transition from fine fibrous structure to columnar grains, eventually leading to a specific orientation. [8]

\section{ELECTRICAL PROPERTIES}

\section{$C-V$ and $G-V$ Characteristics}

The electrical characteristic was carried on both the types of ZT thin films in MIS configuration. Figure 3 shows the $\mathrm{C}-\mathrm{V}$ curve of $10 \mathrm{kHz}$ measured at room temperature on both the types of thin films, which is of high frequency curve. The dielectric constant was measured in the accumulation region and the highly oriented ZT thin films $\left(\varepsilon^{1} \sim 32.8\right)$ exhibited higher dielectric constant than the polycrystalline ZT thin films $\left(\varepsilon^{\prime} \sim 20\right)$. The highly oriented thin films was oriented along $b$ - axis direction and exhibits higher dielectric constant, while the averaging effect of the dielectric constant has been observed in the polycrystalline thin films, eventually resulting in the decrease of the dielectric constant. [9]

The $\mathrm{C}-\mathrm{V}$ curve obtained at frequencies of $1 \mathrm{kHz}$ and higher was of high frequency curve with minimum capacitance at the inversion region, because of the total capacitance arising from the oxide and inversion capacitance in series. The minority carriers do not respond at frequencies higher than $1 \mathrm{kHz}$ and eventually the inversion layer doesn't respond. The response time of the inversion layer is very long ( $0.01-1 \mathrm{sec}$ for $\mathrm{p}-$ type $\mathrm{Si}$ ), when compared to the applied ac signal voltage at the room temperature. Hence the $\mathrm{C}-\mathrm{V}$ measurements was carried at elevated temperatures where the generation of minority carriers is more rapid so as to equilibrate to the applied ac signal voltage. [10]
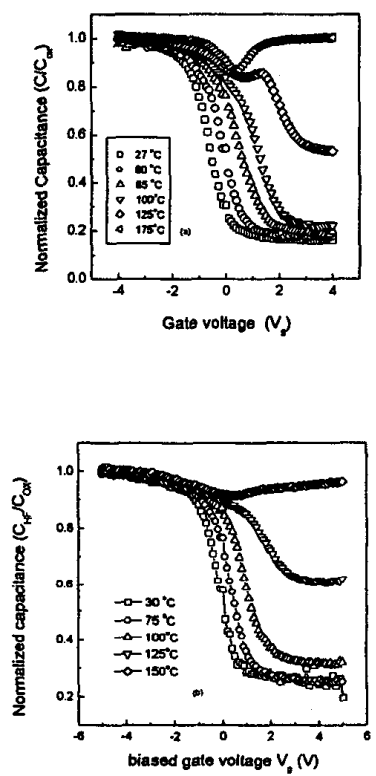

Figure 2. a) $\mathrm{C}-\mathrm{V}$ of the highly oriented and b) polycyrstalline $\mathrm{ZrTiO}_{4}$ thin films

In the case of the highly oriented ZT thin films, the transition frequency takes place at $135^{\circ} \mathrm{C}$, while for the polycrystalline ZT thin films is $141^{\circ} \mathrm{C}$ at $10 \mathrm{kHz}$ as shown in the figure $2 \mathrm{a}$ and figure $2 \mathrm{~b}$. The transition frequency takes place at higher temperature for polycrystalline thin films than highly oriented thin films. The plot of the transition frequency versus reciprocal of the temperature $(1000 / \mathrm{T})$ gives an activation energy of approximately half of the energy band gap in both highly oriented $\left(E_{a}=0.55 \mathrm{eV}\right)$ and polycrystalline $Z T$ thin films $\left(E_{a}=0.57 \mathrm{eV}\right)$. This activation energy obtained in both the types of ZT thin films is 
proportional to half of the silicon band gap energy i.e. activation energy $\left(E_{a}\right)$ is proportional to $n_{i}$. This confirms the generation and recombination of the minority carriers taking place in the depletion layer, till the temperature at which the transition frequency takes place. This process equilibrates the minority carriers in the inversion region with the applied ac signal, which is due to the temperature effect.

The interface states arise due to the dangling bonds or unsaturated bonds at the interface of $\mathrm{Si}$ and $\mathrm{ZrTiO}_{4}$ thin films. Density of the interface states was calculated using the high frequency capacitance method

$D_{i t}=\frac{C_{o x}}{q}\left(\frac{d V_{g}}{d \phi_{s}}-1\right)-\frac{C_{s}}{q}$

with $\phi_{s}$ as surface potential, $\mathrm{C}_{\mathrm{ox}}$ is the oxide capacitance, $V_{g}$ is the gate voltage and $C_{s}$ is the capacitance due to the interface states. [10] The mapping of the energy band gap of Si with the density of interface states is shown in figure 3 . The calculated interface states were found to be higher in polycrystalline thin films than highly oriented ZT thin films. In our case the polycrystalline thin films were ex - situ annealed and the presence of polycrystalline nature suggests that the density of interface states is more than the highly oriented ZT thin films.

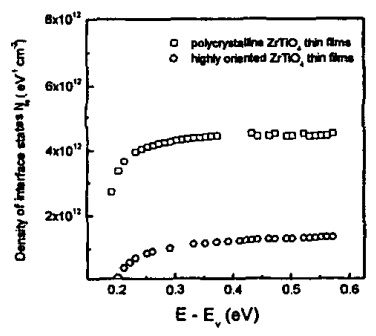

Fig.3. Density of interface states with energy band gap

The conductance - voltage characteristics of both the types of the types of thin films at elevated temperatures. The response of the minority carriers is observed at the transition temperature and above, it is due to the generation - recombination of charges taking place in the depletion region till the transition temperature and above the transition temperature the diffusion process starts dominating with an activation energy equal to $n_{i}{ }^{2}$ as suggested by Nicollian and Goetzberger. [10]

The conductance - voltage had been measured for both the types of thin films. The arrhennius plot of conductance versus $1 / T$ was done till the transition temperature and the activation energy calculated was $0.54 \mathrm{eV}$ and $0.545 \mathrm{eV}$ for highly oriented and polycrystalline thin film which is close to half of the energy band gap of $\mathrm{Si}$. The obtained activation energy and the generation - recombination $\left(G_{g r}\right)$ dominance is in accordance with the $\mathrm{C}-\mathrm{V}$ measurements. The detailed discussion is given elsewhere. [9]

\section{DC leakage characteristics}

An understanding of microstructure correlation with the dc leakage current characteristics on both the types of highly oriented and polycrystalline ZT thin films were analysed in the accumulation region of the MOS capacitor.

\section{Schottky and Poole - Frenkel mechanism}

The highly oriented $\mathrm{ZrTiO}_{4}$ thin films have higher leakage currents than the polycrystalline ZT thin films as shown in figure 4 . The dc leakage current for polycrystalline and highly oriented ZT thin films is $7.2 \times 10^{-9} \mathrm{~A}$ and $1.4 \times 10^{-8} \mathrm{~A}$ respectively, at an electric field of $50 \mathrm{kV} / \mathrm{cm}$ and is shown in figure 5 . For both the types of ZT thin films, due to their large thickness $(150 \mathrm{~nm}$ to $300 \mathrm{~nm})$, tunneling mechanisms cannot be invoked. The Space charge conduction, ionic conduction and schottky conduction mechanism doesn't agree well for both the types of ZT thin films. [11,12]

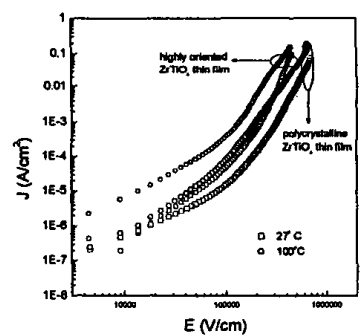

Fig. 4. Leakage currents versus electric field of highly oriented and polycrystalline ZT thin films

The Poole - Frenkel effect predicts a field dependent behavior of the form

$J=C E \exp \left(-\frac{q \phi_{0}}{k_{\mathrm{B}} T}\right) \exp \left(\frac{\beta_{\mathrm{p} f}}{\mathrm{rk}_{\mathrm{B}} \mathrm{T}} \mathrm{E}^{\frac{1}{2}}\right)(7)$
with $\mathrm{C}$ as a constant and
$\beta_{\mathrm{pf}}=\left(\frac{\mathrm{q}^{3}}{\pi \varepsilon_{\mathrm{o}} \mathrm{K}_{\mathrm{T}}}\right)^{1 / 2}$,coefficient $\mathrm{r}$ is taking on the account the influence of the trapping or acceptor centers $(1 \leq r \leq 2)$ with $r=1$ and $r=2$ denoting the normal and 


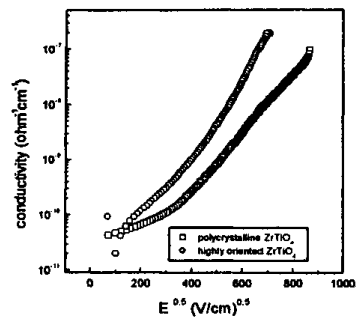

Fig.5. Conductivity versus electric field of both the types of ZT thin films

modified Poole - Frenkel effect respectively. Figure shows the plot of $\ln \sigma v s . E^{1 / 2}$ leads to a straight line and the refractive index calculated from the experimental curves agrees well with the $\mathbf{n}_{Z T}$ for the both the highly oriented $(n \sim 2.1)$ and polycrystalline ZT $(n \sim 2.6)$ thin films. The normal Poole - Frenkel mechanism, which describes the bulk limited charge transport, is identified in both the types of ZT thin films. Both the types of ZT thin films were measured at room temperature, at a given electric field for different thickness and the characteristics of the current density was independent of the films thickness. This strongly suggests that the current is bulk - controlled rather than electrode limited.

\section{DISCUSSION}

The polycrystalline samples exhibited a lower leakage current than the highly oriented ZT thin film as there are presence of electrostatic barriers at the grain grain interfaces which arise from the donor or acceptor like traps localized at the grain boundary. Surface and grain boundaries (GB) introduce additional electronic states in the band gap because of the unsaturated bonds, which arise due to the disruption of the periodical crystal lattice.[13 ] Hence, these grain boundaries act as charge carrier trap sites and essentially create space charge regions around the grain boundaries due to associated charge with the trap sites. Due to presence of the grain boundary space charge layer, which is formed eventually, limits the flow of charges. As the polycrystalline has many numbers of grains and grain boundaries compared highly oriented ZT thin films, the leakage current is higher for highly oriented ZT thin films.

\section{CONCLUSION}

The activation energy calculated based on the capacitance - voltage and conductance - voltage till the transition temperature reveals that the recombination generation mechanism of the charge carriers is prominent. The interface states were calculated by high frequency method and polycrystalline ZT thin films exhibited higher surface states than highly oriented thin films and the leakage current is lower for highly oriented due to the presence of the large number of grain boundaries and interfaces. Both the type of ZT thin films exhibited bulk limited conduction mechanism

\section{ACKNOWLEDGEMENT}

One of the authors wish to thank CSIR, India for the graduate fellowship.

\section{REFERENCES}

[1] G.D.Wilk, R.M.Wallace, J.M.Anthony., " High k gate dielectrics: current status and material propeties considerations" , J.Appl.Phys. 89 (10), 5243 (2001)

[2] K.Wakino, T.Nishikawa, Y.Ishikawa and H.Tamura, "Dielectric resonator materials and applications for mobile communications $\mathrm{Br}$. Ceram. Trans. J. 89 (2), 39 (1990)

[4] P.Bhattacharya, T.Komeda, K.Park and Y.Nishioka., " Comparitive study of amorphous and crystalline BST thin films deposited by laser ablation", Jpn. J.Appl. Phys., 32, 4103 (1993)

[5] S.A.Campbell, H.S.Kim, D.C.Gilmer, B.He, T.Ma and W.L.Gladfelter, "BST dielectrics for future stacked - capacitor DRAM", IBM J.Res.Dev. 43, 383 (1999)

[6] Hiroshi Tamura, "Microwave loss quality of $\left(\mathrm{Zr}_{0.8}, \mathrm{Sn}_{0.2}\right) \mathrm{TiO}_{4}$ ”, American Ceramic society Bulletin , 73 (10), 93, 1994

[7] P.Victor, J.Nagaraju and S.B.Krupanidhi "Analysis of electrical behaviour in highly oriented and polycrystalline $\mathrm{ZrTiO}_{4}$ thin films on Si substrate", J. App. Phys. (communicated 2002)

[8] J.A.Thorton, Annu. Rev. Mater. Sci. 7, 239 (1997)

[9] P.Victor, S.S.N.Bharadwaja, J.Nagaraju and S.B.Krupanidhi., " " solid state comm., 120 , $379(2001)$

[10] E.H.Nicollian and J.R. Brews., MOS (Metal Oxide Semiconductor) Physics and Technology., Wiley NY, 1982

[11] M.A.Lampert and P.Mark., " Current injection in solids", Academic Press, New York 1970

[12] S.M.Sze., " Physics of Semiconductor devices", Wiley publications.

[13] H.Hu and S.B.Krupanidhi, " current - voltage characterisitcs of fine grained PZT thin films", J. Mater. Res., 9, 1484 (1994) 